04

\title{
Экспериментальное исследование рабочего процесса в жидкостных ракетных двигателях с использованием электрофизического метода диагностики
}

\author{
(C) А.Н. Бобров, ${ }^{1}$ А.В. Рудинский, ${ }^{1,2, \uparrow}$ Н.М. Пушкин, ${ }^{3}$ Д.Б. Сафонова, ${ }^{1,2}$ Д.А. Ягодников ${ }^{1}$ \\ ${ }^{1}$ Московский государственный технический университет им. Н.Э. Баумана, \\ 105005 Москва, Россия \\ ${ }^{2}$ Центральный институт авиационного моторостроения им. П.И. Баранова, \\ 111116 Москва, Россия \\ ${ }^{3}$ Научно-производственное объединение измерительной техники, \\ 141070 Королев, Московская обл., Россия \\ ฯ e-mail: alex_rudinskiy@mail.ru \\ Поступило в Редакцию 4 января 2020 г. \\ В окончательной редакции 24 февраля 2020 г. \\ Принято к публикации 26 февраля 2020 г.
}

\begin{abstract}
Проведено экспериментальное исследование собственного электромагнитного поля, генерируемого низкотемпературной плазмой продуктов сгорания топлива модельного жидкостного ракетного двигателя. Компонентами топлива являлись газообразный кислород и этиловый спирт. При моделировании аварийных ситуаций в работе модельного ракетного двигателя зарегистрированы напряженности собственного электромагнитного поля, создаваемого ионизированными продуктами сгорания топлива. Установлена линейная зависимость напряженности собственного магнитного поля от давления в камере сгорания в диапазоне $2.2-3.5 \mathrm{MPa}$. Показана возможность использования бесконтактных электрофизических методов для диагностики рабочего процесса в энергосиловых установках летательных аппаратов при использовании в качестве „полезного“ сигнала значения напряженности магнитного поля.
\end{abstract}

Ключевые слова: горение, электрический заряд, продукты сгорания, собственное электрическое и магнитное поле, напряженность, диагностика.

DOI: $10.21883 / J T F .2020 .08 .49538 .1-20$

\section{Введение}

Одно из основных направлений создания перспективных двигательных установок (ДУ) в изделиях ракетнокосмической техники связано с повышением их надежности, в том числе за счет разработки высокоэффективных методов и средств диагностики, необходимых для реализации лучших тягово-экономических характеристик ДУ (тяга, удельный импульс и др.) при наименьших экономических затратах. Традиционные методы исследования характеристик рабочего процесса в камерах сгорания (КС) ДУ не всегда эффективны, особенно в случае необходимости диагностики качества физикохимических процессов, протекающих в объеме КС, и практически не пригодны для создания системы аварийной защиты, мгновенно реагирующей на изменение рабочего процесса. Указанные недостатки могут быть устранены внедрением новых диагностических методов ДУ, основанных на регистрации электрофизических характеристик рабочего процесса [1]. Предпосылкой данного метода диагностики является то, что процессы горения большинства встречающихся на практике топливных композиций ракетных и воздушно-реактивных двигателей протекают при достаточно высокой температуре в камере сгорания, что обусловливает наличие в продуктах сгорания (ПС) заряженных частиц: электро- нов, положительных и отрицательных ионов, заряженных частиц сажи $[2,3]$ и частиц твердого топлива [4].

В связи с этим, начиная с 70-х годов XX века, проводятся поисковые экспериментальные и теоретические исследования по определению электрофизических характеристик высокоэнтальпийных потоков продуктов сгорания реактивных и ракетных двигателей [5]. Так проведенные измерения электростатических сигналов в выхлопе турбовентиляторного двигателя TF41 позволили разработать основные принципы и схемные решения системы технической диагностики и определить критический износ элементов ДУ [6].

В работах [7-9] объектами исследования являлись газотурбинные двигатели (ГТД), модельные и натурные ракетные двигатели твердого топлива (РДТТ) и жидкостные ракетные двигатели (ЖРД). Измерения электрофизических параметров (пульсации концентраций ионов и твердых заряженных частиц в струе ГТД и РДТТ, электрический ток вдоль заземленной стенки сопла и заряд в затопленном пространстве струи ЖРД и РДТТ, потенциал зонда на входе в ГТД и др.) производились с помощью электростатических зондов и систем цилиндрических электродов, которые требуют непосредственного контакта с высокотемпературным газом (датчикизонды) или достаточно массивны (система цилиндри- 
ческих электродов) для использования на двигателях больших тяг. Экспериментальные работы [7-10] показали принципиальную возможность проведения электростатической диагностики авиационных двигателей и основывались на регистрации потенциала, напряженности и конвективного тока в зависимости от режимных параметров двигателя. Однако нестационарное движение ионов, электронов и заряженных частиц в струе может вызывать появление не только электрических полей, но и индуцировать магнитное поле, напряженность которого связана со скоростью и концентрацией ионов в струе. Кроме того, применительно к ракетным двигателям, температуры в камере сгорания которых достигают более высоких значений по сравнению с авиационными $\left(T_{c h}>3500 \mathrm{~K}\right)$, степень ионизации продуктов сгорания выше, что приводит к интенсификации электромагнитного поля. В условиях огневых испытаний ЖРД электрофизическая диагностика может быть проведена с помощью специальных датчиков, установленных вблизи среза сопла.

Таким образом, цель настоящей работы состояла в экспериментальном определении параметров собственного электромагнитного поля, генерируемого высокоэнтальпийным потоком ПС топлива этиловый спирт+газообразный кислород при моделировании аварийных режимов работы модельного ЖРД (далее МЖРД) и обоснование возможности создания системы бесконтактной диагностики рабочего процесса ЖРД.

\section{Оценка электрического заряда в струе продуктов сгорания МЖРД}

В соответствии с результатами термодинамических расчетов в ПС спиртокислородной смеси содержатся следующие основные носители электрического заряда: свободные электроны, положительные ионы $\mathrm{H}_{3} \mathrm{O}^{+}, \mathrm{O}^{+}$, $\mathrm{O}_{2}^{+}, \mathrm{H}^{+}, \mathrm{H}_{3}^{+}, \mathrm{OH}^{+}, \mathrm{H}_{2} \mathrm{O}^{+}, \mathrm{H}_{3} \mathrm{O}^{+}, \mathrm{CO}_{+}, \mathrm{CO}_{2}^{+}, \mathrm{CHO}^{+}$и отрицательные ионы $\mathrm{OH}^{-}, \mathrm{O}_{2}^{-}, \mathrm{O}^{-}, \mathrm{H}^{-}, \mathrm{OH}^{-}, \mathrm{HO}_{2}^{-}$. Наличие в ПС частиц сажи, их дальнейшая электризация и вклад в заряд и электромагнитное поле истекающей струи в настоящей работе не учитывалось.

Для упрощения примем, что при горении топлива образуется лишь один сорт положительно заряженных ионов, концентрации которых изначально равны концентрации свободных электронов в сечении фронта пламени. Далее при движении по тракту КС к сверхзвуковому соплу электроны частично дрейфуют через металлическую стенку камеры сгорания вследствие их большей подвижности по сравнению с положительными „тяжелыми“ ионами, что в результате обусловливает электрический заряд в сверхзвуковой струе, истекающей через сопло.

Распределение концентраций положительно заряженных ионов и электронов в истекающей струе ПС обусловливает образование собственного электрического поля и индуцированного магнитного поля, параметры которых описываются системой уравнениями Максвелла [10]. Применив метод размерностей к данной системе уравнений, можно оценить уровень напряженности магнитного поля (НМП), создаваемого электрическим зарядом в струе продуктов сгорания, истекающих из сопла МЖРД

$$
H \approx\langle H\rangle=\Omega q_{v}\left\langle v_{a}\right\rangle D_{a}=\Omega e \cdot n\left\langle v_{a}\right\rangle D_{a},
$$

где $\Omega$ - коэффициент пропорциональности, $D_{a}-$ диаметр выходного сечения сопла МЖРД, $H-$ индуцированное магнитное поле, $q_{v}$ - плотность заряда в объеме струи $V$, обусловленная наличием заряженных частиц (электронов и положительных ионов), $n$ объемная концентрация заряженных частиц, $\left\langle v_{a}\right\rangle-$ скорость продуктов сгорания в выходном сечении сопла МЖРД, осредненная по площади сечения.

\section{Описание экспериментальной установки}

МЖРД состоит из нескольких секций, закрепленных вертикально на стапеле (рис. 1). Горючее - этиловый спирт с объемной концентрацией 75\%, окислитель газообразный кислород. Выбор данного топлива обусловлен тем, что при его горении температура в КС достигает значений до $3200 \mathrm{~K}$. Это обеспечивает ионизацию продуктов сгорания, достаточную для проявления газовым потоком, истекающим из сопла, электрофизических свойств. Регистрация электромагнитного поля выхлопной струи ПС МЖРД осуществлялась с помощью датчика магнитного (ДМП) поля, расположение которого показано на рис. 2. Конструкция камеры модельного ЖРД, как в компоновке с охлаждаемым соплом, так и с разгораемым вкладышем имела заземление.

ДМП разработан в „НПО Измерительной техники“ и представляет собой прямой соленоид в корпусе. Технические характеристики ДМП приведены в табл. 1.

Пространственное положение ДМП по отношению к потоку ПС выбиралось таким, чтобы регистрируемый

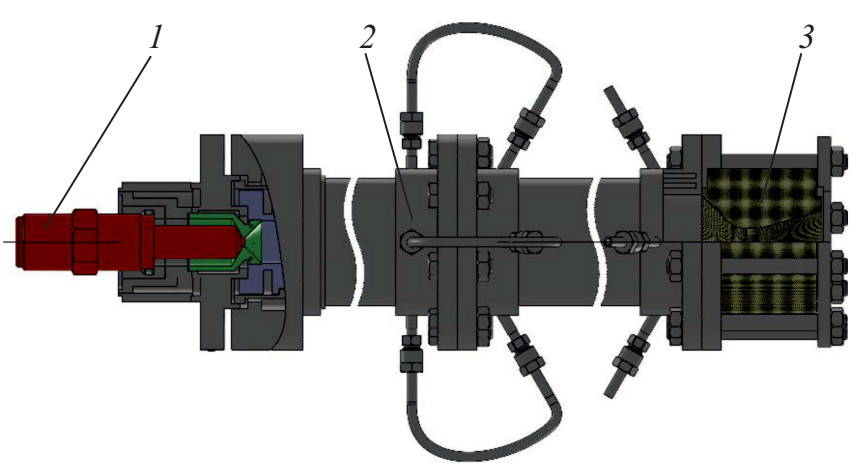

Рис. 1. Схема кислородно-спиртового МЖРД; $1-$ свеча зажигания, 2 - камера сгорания, 3 - сопло (вкладыш из стеклопластика, композита или металлическая секция, охлаждаемая водой). 


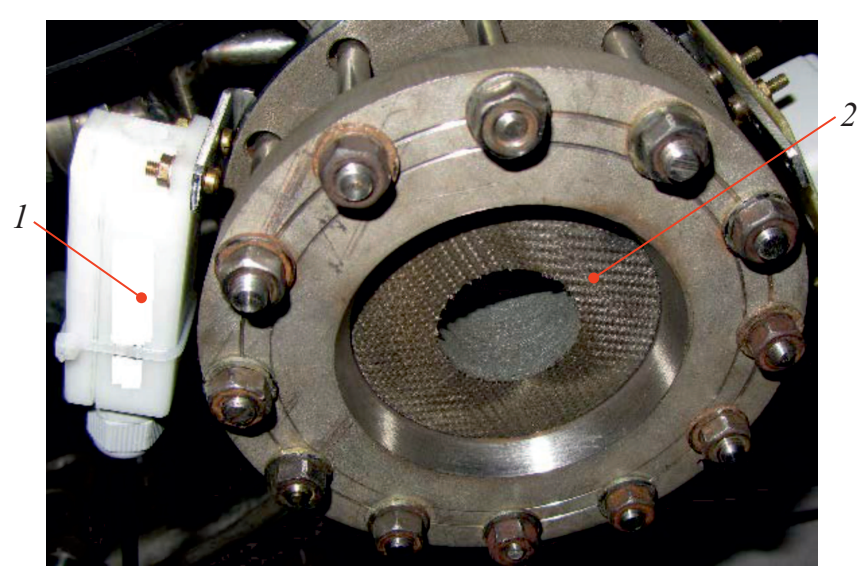

Рис. 2. Расположение ДМП на сопле МЖРД; 1 - датчик магнитного поля, 2 - сопло.

Таблица 1. Технические характеристики ДМП

\begin{tabular}{l|c}
\hline Наименование & Значение \\
\hline $\begin{array}{l}\text { Диапазон измерения напряженности } \\
\text { магнитного поля, } \mathrm{A} / \mathrm{m}\end{array}$ & $0.1-100$ \\
\hline $\begin{array}{l}\text { Частотный диапазон измерения } \\
\text { переменного магнитного поля, } \mathrm{kHz}\end{array}$ & $0.01-20$ \\
\hline $\begin{array}{l}\text { Ток потребления, А } \\
\text { Габаритные размеры датчика, } \mathrm{mm}\end{array}$ & $18 \times 30 \times 20$
\end{tabular}

полезный сигнал был наибольшим. Это достигалось путем предварительных градуировок ДМП, а также на основе электрофизической картины истечения ПС из сопла. Предполагается, что вектор движения заряженных частиц в струе совпадает с направлением потока, следовательно, линии напряженности магнитного поля, генерируемого движущимся зарядом, расположены в радиальной плоскости и представляют собой окружности, соосные потоку ПС. Поэтому ДМП закреплялся снаружи камеры в районе среза сопла таким образом, чтобы вектор напряженности магнитного поля оказался соосным оси обмотке соленоида датчика.

Схема подачи компонентов топлива в камеру МЖРД с регистрацией электромагнитного поля представлена на рис. 3. Газообразный кислород из баллона 1 поступает через редуктор 2. В магистрали кислорода измеряется перепад давления на редукторе и полное давление 3 перед мерным соплом 4. После подачи сигнала на электропневмоклапан 5 кислород поступает в смесительную головку и далее в КС МЖРД 6. После открытия вентиля 7 этиловый спирт поступает из бака 8 через турбинный преобразователь расхода 10 в КС. Постоянство расходов газообразного кислорода и этилового спирта и, следовательно, значения массового соотношения компонентов, характеризуемого коэффициентом избытка окислителя $\alpha$, в течение эксперимента с раз- гаром критического сечения сопла обеспечивается установкой на магистралях компонентов сверхзвукового 4 и кавитационного сопел 9 соответственно. Воспламенение компонентов топлива в КС осуществляется электрической свечой, напряжение на которую подается от высоковольтного генератора. Система измерения параметров экспериментальной установки с МЖРД реализована на базе измерительного комплекса МIC-300 разработки АО „НПП Мера“. Медленноменяющиеся параметры с первичных измерительных преобразователей давления, перепада давлений и турбинного преобразователя расхода регистрировались с частотой опроса $100 \mathrm{~Hz}$ и предельной погрешностью $\pm 0.3 \%$, а быстроменяющиеся характеристики магнитного поля - c частотой опроса $50 \mathrm{kHz}$. Погрешность косвенного измерения коэффициента избытка окислителя составила $\pm 2.2 \%$.

Регистрация сигнала с ДМП 11 , создаваемого зарядом в сверхзвуковой струе 12 , происходила следующим образом. Сигнал (напряжение) с ДМП поступает на промежуточные усилители и через плату сопряжения 13 регистрируется в памяти системы измерения 14. Преобразование сигнала с ДМП, полученного в виде электрического напряжения, в напряженность магнитного поля $(\mathrm{A} / \mathrm{m})$ производилось с помощью градуировочной зависимости.

Градуировка ДМП осуществлялась путем измерения напряженности магнитного поля внутри соленоида длиной $300 \mathrm{~mm}$ и диаметром $30 \mathrm{~mm}$. ДМП располагался внутри соленоида примерно на его середине. Зависимость напряжения, генерируемого ДМП при градуировке, удовлетворительно аппроксимировалась линейной функцией

$$
U=0.0023 \cdot H+0.0834,
$$

где $H[\mathrm{~A} / \mathrm{m}], U[\mathrm{~V}]$.

Основная погрешность измерения при градуировке составила $3 \%$. Погрешность измерения магнитных ве-

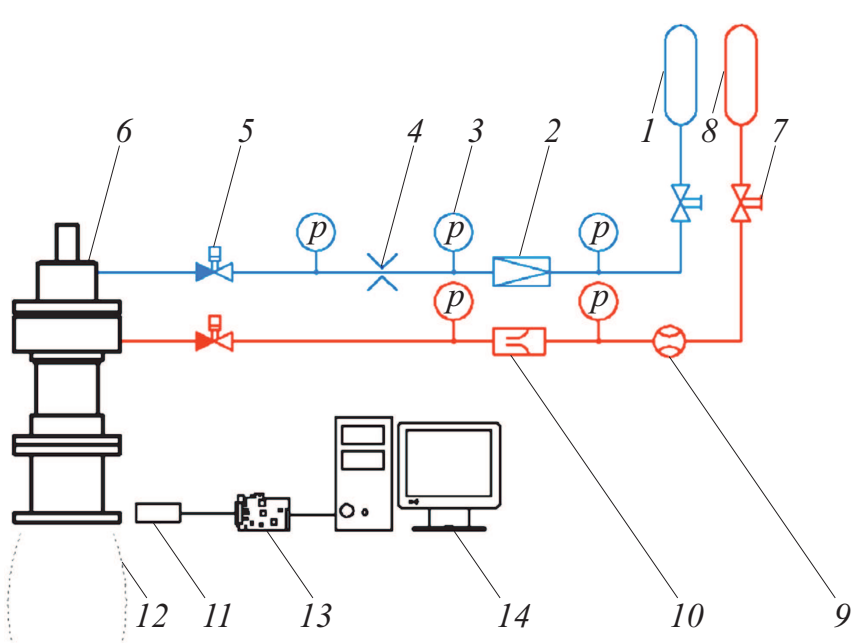

Рис. 3. Схема экспериментальной установки (пояснения в тексте). 
личин при проведении огневого испытания с учетом дополнительных воздействий, таких как нагрев, вибрации, акустика и др. составила не более $5 \%$.

\section{Результаты экспериментальных исследований}

Основной задачей экспериментального исследования являлось моделирование возникновения аномальных режимов работы МЖРД, которые могут быть обусловлены разрушением элементов системы подачи топлива в результате прогара лопаток турбины турбонасосного агрегата, смесительной головки, огневой стенки КС, критического сечения и расширяющейся части сопла.

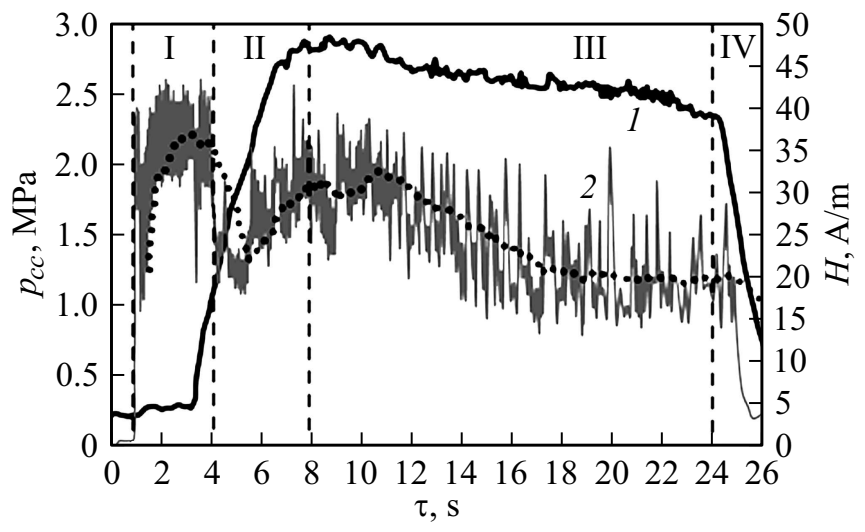

Рис. 4. Осциллограмма режимных и электрофизических параметров. Испытание при дросселировании массового расхода кислорода в КС; $p_{c c}$ - давление в КС, $H-$ напряженность магнитного поля. I - работа агрегата зажигания, II выход МЖРД на режим $p_{c c 1}=2.9 \mathrm{MPa}(\alpha \approx 0.75)$, III дросселирование расхода кислорода и уменьшение давления до $p_{c c 2}=2.5 \mathrm{MPa}(\alpha \approx 0.74), \mathrm{IV}$ - останов МЖРД.

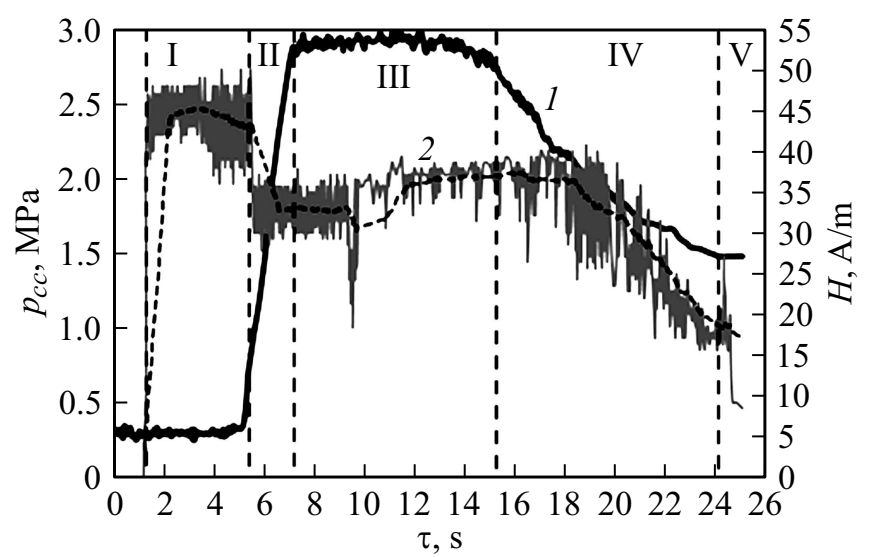

Рис. 5. Осциллограмма режимных и электрофизических параметров. Испытание при разгаре вкладыша критического сечения из композита; $p_{c c}-$ давление в КС, $H-$ напряженность магнитного поля. I - работа агрегата зажигания, II - выход МЖРД на режим $p_{c c 1} \approx 3 \mathrm{MPa}(\alpha \approx 0.74)$, III - работа ЖРД на режиме, IV - разгар вкладыша критического сечения $p_{c c 2} \approx 1.5 \mathrm{MPa}, \mathrm{V}$ - останов МЖРД.

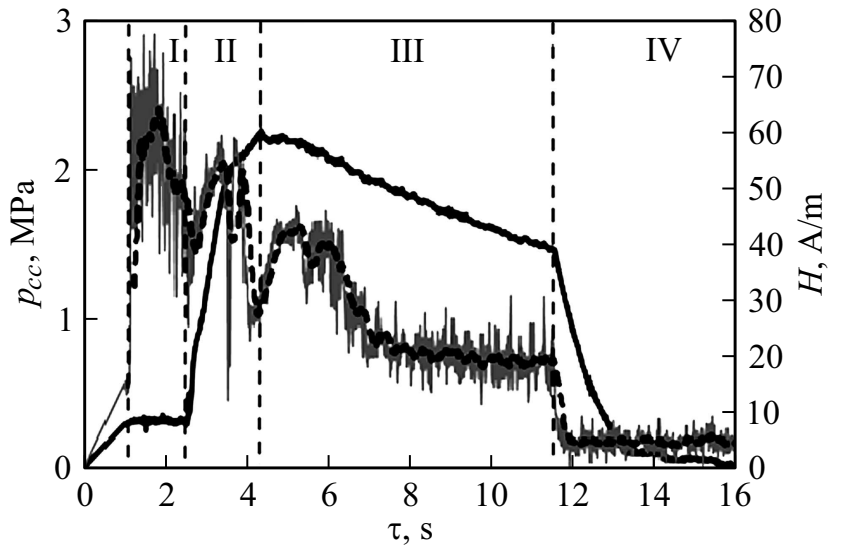

Рис. 6. Осциллограмма режимных и электрофизических параметров. Испытание при разгаре вкладыша критического сечения из стеклопластика; $p_{c c}$ - давление в КС, $H-$ напряженность магнитного поля. $I-$ работа агрегата зажигания, II - выход ЖРД на режим $p_{c c 1} \approx 2.2 \mathrm{MPa}(\alpha \approx 0.91)$, III - разгар вкладыша критического сечения $p_{c c 2} \approx 1.4 \mathrm{MPa}$ $(\alpha \approx 0.92)$, IV - останов ЖРД.

В первой серии огневых стендовых испытаний (ОСИ) этот процесс моделировался при помощи изменения массового расхода газообразного кислорода, подаваемого в КС. Геометрия сопла не изменялась в процессе ОСИ. Сопло изготовлено из жаропрочной стали и охлаждалось водой. Диаметр критического сечения сопла $d_{\mathrm{cr}}=18 \mathrm{~mm}$, диаметр КС $d_{c c}=60 \mathrm{kjmm}$, диаметр среза сопла $d_{a}=40 \mathrm{~mm}$. Диапазоны изменения режимных параметров модельного ЖРД: давление в КС 1.4-3 МРа и коэффициент избытка окислителя 0.75-0.9 выбирались из условия достижения объемных концентраций электронов и ионов, необходимых для проявления электрофизических свойств потока. Верхнее значение давления в КС ограничивалось прочностью камеры.

Из осциллограммы пуска (рис. 4) следует, что по мере уменьшения давления в КС $p_{c c}=2.9-2.5 \mathrm{MPa}$ происходит монотонное уменьшение уровня НМП $H=40-25 \mathrm{~A} / \mathrm{m}$.

Данный результат можно объяснить следующим образом. При уменьшении давления в КС МЖРД плотность продуктов сгорания также уменьшается в соответствии с уравнением неразрывности. При этом, в соответствии с уравнением (1), суммарный электрический заряд пропорционален объемной концентрации заряженных частиц, образовавшихся во фронте горения в камере сгорания. В соответствии с допущениями, принятыми ранее, концентрации электронов и положительных ионов равны в прифронтовой зоне КС. Далее по тракту камеры МЖРД электроны частично дрейфуют через металлическую стенку камеры сгорания вследствие большей подвижности, по сравнению с положительными ионами, что в результате обусловливает электрический заряд в сверхзвуковой струе, истекающей через сопло. 
Таблица 2. Объемные концентрации электронов в продуктах сгорания: этиловый спирт + кислород (газ)

\begin{tabular}{c|c|c|c|c|c}
\hline$p_{c c}, \mathrm{MPa}$ & $\alpha$ & $T_{c}, \mathrm{~K}$ & $n_{e}, \mathrm{~cm}^{-3}$ & $n_{i}^{+}, \mathrm{cm}^{-3}$ & $H, \mathrm{~A} / \mathrm{m}$ \\
\hline 2.9 & 0.75 & 2878.4 & $0.8399 \cdot 10^{11}$ & $1.2280 \cdot 10^{11}$ & 32.5 \\
2.5 & 0.74 & 2703.4 & $0.6418 \cdot 10^{11}$ & $0.9016 \cdot 10^{11}$ & 20.0 \\
3.0 & 0.74 & 2855.1 & $0.7577 \cdot 10^{11}$ & $1.0970 \cdot 10^{11}$ & 36.5 \\
1.5 & 0.74 & 2848.3 & $0.4468 \cdot 10^{11}$ & $0.5898 \cdot 10^{11}$ & 19.0 \\
2.2 & 0.91 & 3051.1 & $1.4550 \cdot 10^{11}$ & $2.4500 \cdot 10^{11}$ & 41.1 \\
1.4 & 0.92 & 3014.0 & $0.8841 \cdot 10^{11}$ & $1.3490 \cdot 10^{11}$ & 19.9
\end{tabular}

Следующим этапом проведения ОСИ являлось исследование влияния разгара сопла на уровень НМП. Сопло в виде вкладыша изготавливалось из композитного материала. Размеры проточного тракта сопла вкладыша сопла идентичны охлаждаемому соплу. Запуск и выход МЖРД на режим (рис. 5) осуществлялся аналогично ОСИ на рис. 4. Отличия появляются в тот момент, когда материал сопла не выдерживает теплового и эрозионного воздействия потока и с $16 \mathrm{~s}$ ОСИ начинается разгар вкладыша, сопровождающийся монотонным падением давления в КС согласно уравнению неразрывности. Одновременно с этим наблюдалось уменьшение уровня сигнала ДМП, которое на останове МЖРД соответствует НМП $\approx 20 \mathrm{~A} / \mathrm{m}$ (рис. 5 , область $\mathrm{V}$ ). Кроме того, осциллограмма пуска свидетельствует об увеличении амплитуды сигнала ДМП, что может быть объяснено выносом из КС заряженных конденсированных частиц, являющихся продуктами термохимического разложения материала вкладыша сопла.

При использовании менее теплостойкого материала вкладыша из стеклотекстолита (рис. 6) разгар критического сечения сопла начинается до выхода на планируемый режим МЖРД по давлению в КС $\left(p_{c c} \approx 3 \mathrm{MPa}\right)$. Поскольку осциллограмма на рис. 6 иллюстрирует постоянство коэффициента избытка окислителя $(\alpha \approx 0.91)$ в течение интервала времени, соответствующего разгару вкладыша (рис. 6, область III), можно утверждать, что изменение уровня НМП непосредственно обусловлено падением давления в КС и увеличением скорости потока в КС вследствие увеличения площади критического сечения в результате разгара.

В табл. 2 приведены расчетные равновесные концентрации электронов и положительных ионов в ПС. Исходными данными для расчета концентраций в табл. 2 принимались режимы работы МЖРД в соответствии с данными испытаний на рис. 4 (в начале и в конце режима III), рис. 5 (в начале и в конце режима IV), pис. 6 (в начале и в конце режима III). Так изменение расчетных объемных концентраций практически прямо пропорционально изменению уровня НМП, зарегистрированному в испытаниях на рис. 4-6, что подтверждает зависимость (1). Следует отметить, что во всех испытаниях ДМП регистрирует повышенные значения НМП (до $65 \mathrm{~A} / \mathrm{m}$ ) при работе электроискровой систе-

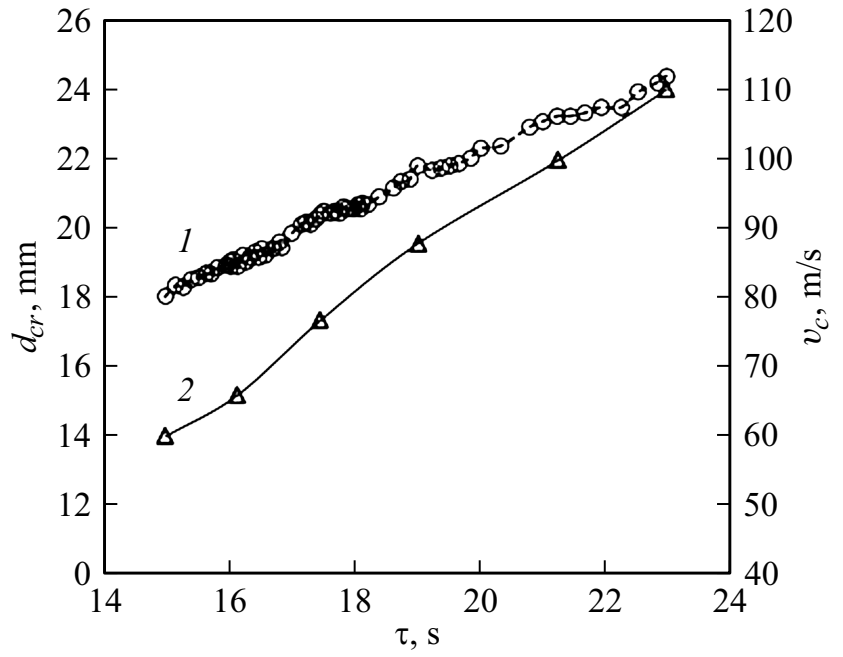

Рис. 7. Осциллограмма изменения эквивалентного диаметра критического сечения сопла и скорости газа в КС при разгаре вкладыша из стеклопластика; $d_{c r}-$ эквивалентный диаметр критического сечения сопла, $v_{c}-$ скорость газа в КС.

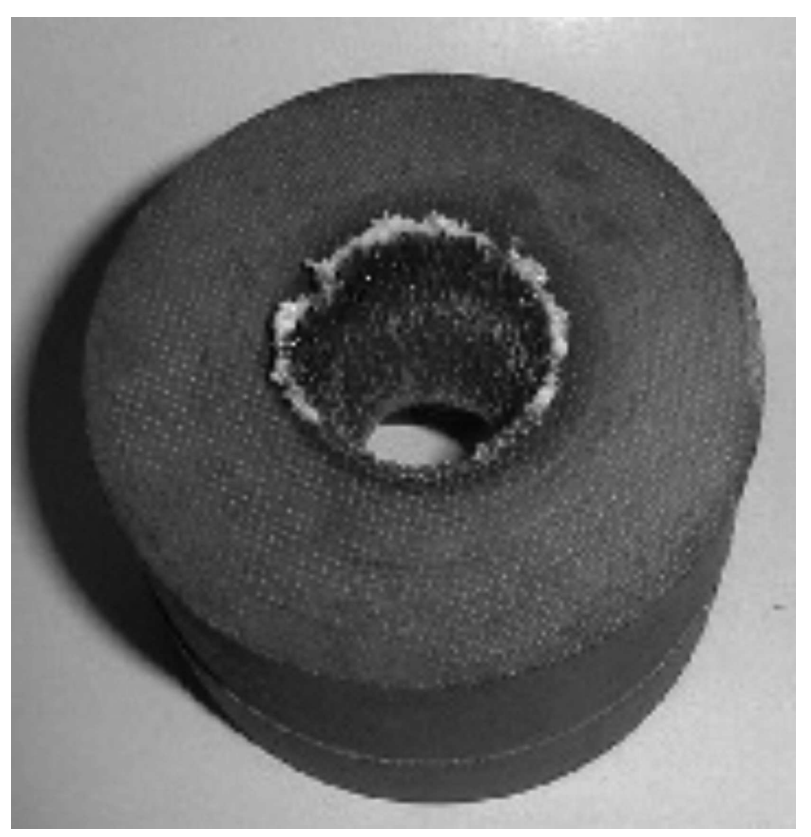

Рис. 8. Вкладыш критического сечения из композитного материала после огневого стендового испытания.

мы зажигания (режим I). Расчетная оценка ионизации газообразного кислорода и уровня НМП в результате воздействия свечи, предшествующая воспламенению топлива, в настоящей работе не проводилась.

Проведем оценки эквивалентного диаметра критического сечения при разгаре сопла. Из уравнения неразрывности следует, что в процессе разгара критического сечения сопла при постоянном массовом расходе топлива должно происходить падение давления в КС. Следовательно, по уменьшению давления в КС в результате разгара вкладыша (рис. 6) можно оценить увеличение 


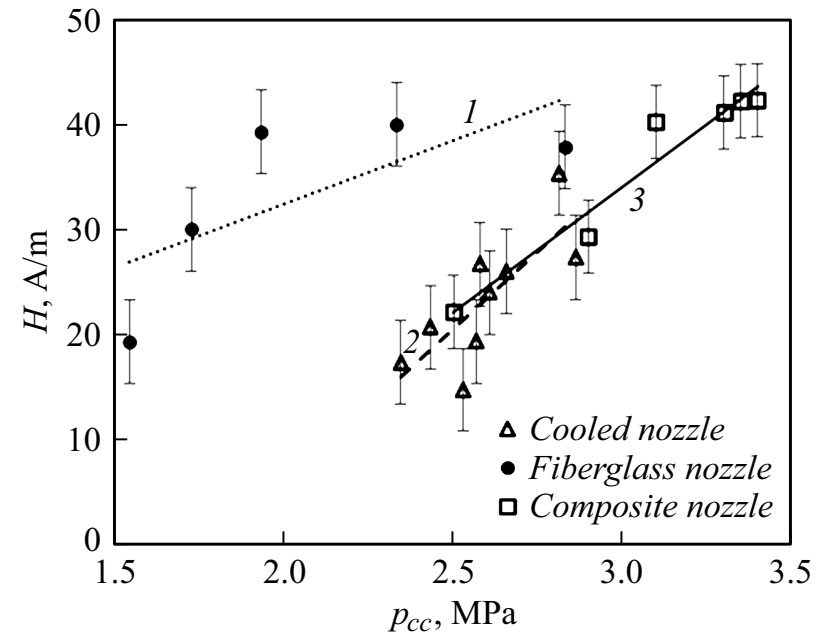

Рис. 9. Напряженность собственного магнитного поля ионизированного потока в зависимости от давления в КС при моделировании аварийных ситуаций в работе МЖРД; 1-3линейная аппроксимация.

эквивалентного диаметра критического сечения сопла по зависимости

$$
\left.\left.d_{(} \operatorname{cr} i\right)=d_{(} \operatorname{cr} 0\right) \sqrt{\frac{p_{c c} 0}{p_{c c i}}},
$$

где $p_{c c} 0, d_{c r 0}-$ давление в КС и диаметр критического сечения до разгара, $p_{c c i}$ - давление в КС, соответствующее $i$-му времени разгара.

Для интервалов времени, соответствующих разгару сопла (рис. 6, область III), изменение эквивалентного диаметра критического сечения, рассчитанное по выражению (2), приведено на рис. 7. Как видно из рис. 7, при увеличении диаметра критического сечения с 18 до $24 \mathrm{~mm}$ при разгаре сопла увеличивается скорость в КС, которая в конце разгара, согласно оценкам, составляла $\sim 110 \mathrm{~m} / \mathrm{s}$. Такое увеличение скорости в КС может являться причиной снижения полноты сгорания топлива. Это, в свою очередь, вызывает снижение температуры в КС и концентрации ионов, что уменьшает уровень НМП. Общий вид вкладыша критического сечения из композитного материала после огневого испытания показан на рис. 8.

Анализ результатов проведенной серии ОСИ при различных давлениях в КС позволил установить зависимость уровня напряженности магнитного поля от давления в КС (рис. 9), которая в диапазоне 2.5-3.5 МРa аппроксимируется линейной функцией

$$
H=23.814 p_{c c}-37.626,
$$

где $H[\mathrm{~A} / \mathrm{m}], p_{c c}[\mathrm{MPa}]$.

Отметим, что качественные характеры зависимостей НМП от давления в КС, полученные при разгаре сопла из композиционного материала и с помощью изменения расхода через КС, удовлетворительно совпадают.
Полученный результат также подтверждает, что значение заряда, определяющего характеристики электромагнитного поля истекающей струи ПС, пропорционально произведению диаметра среза сопла, скорости потока и концентрации ионов в струе согласно зависимости (1). Уменьшение давления в КС и, следовательно, концентрации ионов приводит к уменьшению уровня НМП. Однако уровень НМП, зарегистрированный при разгаре вкладыша из стеклопластика, выше, несмотря на то, что диапазон давлений в КС был меньше. Данный результат может быть объяснен дополнительным зарядом, обусловленным горением частиц стеклопластика с эмиссией электронов и последующей зарядкой таких частиц, что вызвало увеличение регистрируемого уровня НМП.

Таким образом, установлена корреляция между давлением в КС и показаниями ДМП при ОСИ, которая может быть использована как диагностический признак в системе электрофизической диагностики и регулирования ЖРД.

Определенный интерес представляет сравнение полученных значений напряженности собственного магнитного поля ПС, зарегистрированных при огневых испытаниях, с экспериментальными данными других авторов. Так, например, в работе [7], при испытаниях ЖРД измерялся электрический ток между кольцевым электродом, омываемым газовой струей, и заземленным двигателем. Электрический заряд, генерируемый струей, составил до $0.6 \cdot 10^{-6} \mathrm{C}$ в зависимости от времени испытания 0-2 s.

Оценим величину электрического заряда в струе продуктов сгорания по выражению (1). Согласно экспериментальным результатам, полученным в настоящей работе, измеренная напряженность магнитного поля составила $H \approx 30 \mathrm{~A} / \mathrm{m}$, что при расчетной скорости в выходном сечении сопла $v_{a} \approx 2400 \mathrm{~m} / \mathrm{s}$ и диаметре выходного сечения $D_{a}=40 \mathrm{~mm}$ дает величину объемного заряда $Q_{v}=0.313 \mathrm{C} / \mathrm{m}^{3}$. Учитывая, что данные в [7] получены при нахождении кольцевого электрода на расстоянии $L_{0}$ порядка $10 \%$ от диаметра выходного сечения сопла (т.е. $L_{0}=0.1 \cdot D_{a}$ ), электрический заряд будет пропорционален объему цилиндра, составляющего пространство струи до предполагаемого электрода

$$
q=Q_{V} \cdot V_{C y l}=Q_{V} \cdot 0.25 \cdot \pi \cdot D_{a}^{2} \cdot L_{0} \approx 1.6 \cdot 10^{-6} \mathrm{C} .
$$

Полученное значение по порядку величины согласуется с электрическим зарядом $q \approx 0.6 \cdot 10^{-6} \mathrm{C}$ [7], зарегистрированным при испытаниях двигателя с близкими к исследуемому геометрическими и режимными параметрами $\left(D_{a}=40 \mathrm{~mm}, p_{c c}=0.6 \mathrm{MPa}, T_{c}=3150 \mathrm{~K}\right)$.

\section{Заключение}

Резюмируя вышеизложенное, сформулируем следующие основные выводы и практические рекомендации по использованию электрофизических методов контроля и диагностики ЖРД. 
1. Разработана методика экспериментального определения характеристик собственного электромагнитного поля потока при истечении продуктов сгорания кислорода и этилового спирта из сверхзвукового сопла МЖРД, позволившая установить зависимость напряженности магнитного поля от давления в камере сгорания, которая в случае „чистого“ газа удовлетворительно аппроксимируется линейной функцией.

2. При испытаниях МЖРД на стенде получено, что сигнал ДМП зависит от режимных параметров МЖРД $\left(p_{c c}, \alpha\right)$ и от параметров рабочего процесса в КС.

3. При глубоком дросселировании камеры МЖРД уровень НМП изменяется прямо пропорционально давлению в КС.

4. При увеличении площади критического сечения и постоянном расходе через камеру двигателя уровень НМП падает пропорционально давлению в КС. Это объясняется уменьшением полноты сгорания топлива в результате увеличения скорости в КС. Полученный результат может быть использован, например, в системах диагностики и контроля полноты сгорания в воздушнореактивных двигателях, в конструкции которых предусмотрено регулирование критического сечения сопла.

5. В качестве диагностических признаков при разработке бесконтактной системы диагностики и аварийной защиты ЖРД и энергосиловых установок могут использоваться следующие параметры: напряженность собственного электромагнитного поля факела (для контроля соотношения компонентов и полноты сгорания топлива); пульсации электрического потенциала струи, обусловленные электризованными частицами $\kappa$-фазы (для контроля процесса эрозии элементов конструкции ДУ и ранней диагностики отказа).

6. При сравнительном анализе зависимостей уровней напряженности магнитного поля от давления в КС, полученных при уменьшении расхода топлива в КС и при разгаре критического сечения сопла, установлено, что частицы стеклотекстолита повышают заряд струи, что может быть использовано при параметрической диагностике начала разгара сопла.

В заключении отметим, что применение данного метода диагностики в условиях полета летательного аппарата сопряжено с решением ряда важных технических проблем. Например, при полете заземление конструкции двигателя невозможно, и камера двигателя постепенно приобретет отрицательный „плавающий“ потенциал, при котором электронный ток на стенку сопла будет компенсирован ионным током, и в соответствии с теоремой Гаусса, магнитная индукция (напряженность магнитного поля) в точке измерения обратиться в нуль. Однако данный метод применительно к диагностике твердых частиц в потоках ракетных и реактивных двигателей может обладать высокой надежностью и в условиях полета.

\section{Финансирование работы}

Работа выполнена при поддержке Госпрограммы № 0705-2020-0044 фундаментальных исследований лаборатории „Внутрикамерные процессы ракетных и реактивных двигателей“.

\section{Конфликт интересов}

Авторы заявляют, что у них нет конфликта интересов.

\section{Список литературы}

[1] Lawton J., Weinberg F. Electrical Aspects of Combustion. Oxford University Press, Oxford. 1969.

[2] Sorokin A., Arnold F. // Atmospheric Environment. 2004. Vol. 38. P. 2611-2618.

[3] Sorokin A., Vancassel X., Mirabel P. // Atmos. Chem. Phys. 2003. Vol. 3. P. 325-334.

[4] Dunn R.W. // Int. J. Turbo Jet Eng. 1999. Vol. 16. P. 255-262.

[5] Shaeffer J.F., Peng T.C. // AIAA J. Eng. Notes. 1977. P. 429-431.

[6] Robert P. // J. Aircraft. 1978. Vol. 15. N 10. P. 692-695.

[7] Нагель Ю.А. // ЖТФ. 1999. Т. 69. Вып. 8. С. 55-59. [Nagel' Yu.A. // Tech. Phys. 1999. Vol. 44. N 8. P. 918-922.]

[8] Wei Liu, Manguo Huang, Defeng Liu, Yanshan Wang, Meiju Zhang. Design and Test of Sensor for Aircraft Engine Gas Path Debris Electrostatic Monitoring // MATEC Web of Conferences. 2017. Vol. 139. N 00145. P. 1-4.

[9] Yibing Yin, Jing Cai, Hongfu Zuo, Huïie Mao, Yu Fu, Hongsheng Yan. // J. Vibroengineer. 2017. Vol. 19. N 2. P. $967-987$.

[10] Vatazhin A.B., Golentsov D.A., Likhter V.A., Shulgin V.I. // J. Electrostat. 1997. N 40-41. P. 711-716. 